\title{
Improved transition to adult care in youth with type 1 diabetes: a pragmatic clinical trial
}

\author{
Sonia Butalia ${ }^{1,2,3,4}$ (D) Susan G. Crawford ${ }^{5} \cdot$ K. Ashlee McGuire ${ }^{6,7} \cdot$ David K. Dyjur $^{8}$ (D) - Julia R. Mercer ${ }^{9}$ (D) \\ Danièle Pacaud ${ }^{10,11}$
}

Received: 11 August 2020 / Accepted: 4 November 2020 / Published online: 13 January 2021

(C) The Author(s), under exclusive licence to Springer-Verlag GmbH, DE part of Springer Nature 2021

\begin{abstract}
Aims/hypothesis Youth with type 1 diabetes are at high risk for loss to follow-up during the transition from paediatric to adult diabetes care. Our aim was to assess the effect of a communication technology enhanced transition coordinator intervention compared with usual care on clinic attendance among transitioning youth with type 1 diabetes.

Methods In this open label, pragmatic clinical trial of youth with type 1 diabetes, aged 17-18 years, transitioning from paediatric to adult diabetes care, the intervention group received support from a transition coordinator who used communication technology and the control group received usual care. The primary outcome was the proportion of individuals that did not attend at least one routine clinic visit in adult diabetes care within 1 year after transfer. Secondary outcomes included diabetes-related clinical outcomes and quality of life measures.

Results There were no baseline differences in age, sex, $\mathrm{HbA}_{1 \mathrm{c}}$ and number of follow-up visits, emergency department visits and diabetic ketoacidosis admissions in the 1 year prior to transition between the usual care $(n=101)$ and intervention $(n=102)$ groups. In the year following transfer, $47.1 \%$ in the usual care group vs $11.9 \%$ in the intervention group did not attend any outpatient diabetes appointments $(p<0.01)$. There were no differences in glycaemic control or diabetic ketoacidosis post transfer.

Conclusions/interpretation Our intervention was successful in improving clinic attendance among transitioning youth with type 1 diabetes. Importantly, this programme used simple, readily accessible communication technologies, which increases the sustainability and transferability of this strategy.

Trial registration isrctn.org ISRCTN13459962
\end{abstract}

Keywords Pragmatic trial $\cdot$ Transition to adult care $\cdot$ Type 1 diabetes $\cdot$ Youth

Sonia Butalia

sbutalia@ucalgary.ca

1 Division of Endocrinology and Metabolism, Department of Medicine, Cumming School of Medicine, University of Calgary, Calgary, AB, Canada

2 Department of Community Health Sciences, Cumming School of Medicine, University of Calgary, Calgary, AB, Canada

3 O'Brien Institute for Public Health, Cumming School of Medicine, University of Calgary, Calgary, AB, Canada

4 Libin Cardiovascular Institute, Cumming School of Medicine, University of Calgary, Calgary, AB, Canada

5 Alberta Health Services, Calgary, AB, Canada
6 Provincial Primary Health Care, Alberta Health Services, Calgary, AB, Canada

7 Department of Family Medicine, Cumming School of Medicine, University of Calgary, Calgary, AB, Canada

8 Diabetes Centre Calgary, Alberta Health Services, Calgary, AB, Canada

9 Alberta Children's Hospital, Diabetes Clinic, Alberta Health Services, Calgary, AB, Canada

10 Division of Pediatric Endocrinology and Diabetes, Department of Pediatrics, Cumming School of Medicine, University of Calgary, Calgary, AB, Canada

11 Alberta Children's Hospital Research Institute, Cumming School of Medicine, University of Calgary, Calgary, AB, Canada 


\section{Research in context}

\section{What is already known about this subject?}

- During the transition from paediatric to adult care, youth with diabetes are at high risk for loss to follow-up, poor glycaemic control and increased hospitalisations

- Thus far, recent transition trials report mixed results in improving follow-up rates post transfer

\section{What is the key question?}

- Compared with usual care, what is the effect of a communication technology enhanced transition coordinator intervention on clinic attendance among transitioning youth with type 1 diabetes?

\section{What are the new findings?}

- Our communication technology enhanced transition coordinator intervention was successful in improving clinic attendance among transitioning youth with type 1 diabetes

- There was a fourfold reduction in loss to follow-up in adult care

- Although the mean $\mathrm{HbA}_{1 c}$ did not improve, the proportion of individuals with a mean $\mathrm{HbA}_{1 \mathrm{c}}>9 \%$ was lower post transition in the intervention group

\section{How might this impact on clinical practice in the foreseeable future?}

- This intervention used a non-medical transition coordinator with simple, readily accessible communication technologies, thus increasing the sustainability and transferability of this strategy

\section{Abbreviations \\ ACR Albumin/creatinine ratio \\ DAD Discharge Abstract Database \\ NACRS National Ambulatory Care Reporting System \\ PHN Personal Health Number}

\section{Introduction}

Type 1 diabetes is one of the most common chronic childhood conditions. Transition from paediatric to adult diabetes care is a crucial window for adult health determination and has long been recognised as a time of vulnerability [1-3]. Youth with type 1 diabetes who have inadequate transition have up to $60 \%$ loss to medical follow-up and are at risk of increased hospitalisations [1-3]. This period is also associated with poor self-care behaviours and mental health issues [4, 5]. Unfortunately, current healthcare services are failing to meet the needs of transitioning youth with type 1 diabetes [4-8]. There is ever the need to improve transition care as the incidence and prevalence of type 1 diabetes is increasing $[9,10]$.

During transition to adult care, youth with diabetes have multiple concurrent competing priorities (school, relationships, finances, etc.) in addition to their medical needs. There are also changes in their medical care including changes in their diabetes care providers, clinic location and care delivery and style. Together, these issues contribute to disruptions and disengagement with adult diabetes care. In general, follow-up rates post transfer to adult diabetes care are poor
[11-13]. Recent trials have attempted to improve post-transfer follow-up rates, with mixed results. Two studies did not demonstrate improved follow-up rates while one study reported improved clinic attendance during their intervention but not in subsequent follow-up [12-14].

The development of a communication technology enhanced transition coordinator intervention resulted from the following: consultations with local stakeholders including paediatric and adult endocrinologists, nurses, dieticians, psychologists and healthcare managers; transition programmes across the country; and a literature review. Most importantly, input from patients and their families was obtained through focus groups [8]. The objective of this study was to assess the effect of this intervention on clinic attendance among youth with type 1 diabetes transitioning from paediatric to adult diabetes care.

\section{Methods}

Study design This pragmatic clinical trial was an open label, non-randomised clinical trial conducted with seven paediatric endocrinologists within one tertiary care paediatric hospital and eight adult endocrinologists within community sites, an adult community hospital and a regional diabetes centre in Alberta, Canada. We purposely selected this study design to assess whether this intervention works as there is an imminent need to demonstrate real-world effectiveness, rather than efficacy in a highly selected group not representative of our target 
population [15]. Further, using an individual recruitment design would likely have led to a selection bias as the most vulnerable youth who would most benefit from a transition coordinator would have been less likely to enrol in a study. Therefore, for the purpose of this study, to avoid this selection bias, the communication transition coordinator's role was established as 'standard of care for all patients' attending paediatric clinics for the period of the intervention. The control (usual care) group was a group of youth not exposed to the communication transition coordinator for a period equivalent to the intervention prior to the start of the intervention. This study design met the criteria for a quality improvement project and ethics allowed for us to include the data from all individuals meeting the inclusion criteria for the two designated groups, without individual consent, avoiding selection bias. Informed consent was obtained for the questionnaire components of the study. We used the Strengthening the Reporting of Observational Studies in Epidemiology statement for the reporting framework of this study [16].

Enrolment Individuals were included if they fulfilled all the following requirements: (1) had a diagnosis of type 1 diabetes according to the ADA for at least the last 6 months; (2) were aged 17-18 years; (3) had been seen by their paediatric endocrinologist in the last 12 months; (4) were in the last year of paediatric care and transferring to an adult care site in the city within the next year; and (5) had a Personal Health Number (PHN) for data linkage [17]. Both usual care and intervention group participants were enrolled from the same paediatric clinics. The usual care group were enrolled from 1 January 2013 to 31 May 2014 and intervention group participants were enrolled from 1 April 2015 to 31 August 2016 with a 1 year washout period between enrolment of groups. There were no other changes to transition procedures or processes across the study period, as we engaged clinical staff and administrators from paediatric and adult centres throughout our programme of work.

Intervention Both usual care and intervention groups received routine diabetes care as per national guidelines [18]. The transition process started at age 14 years with discussions during clinic visits with youth and families around increased autonomy, self-care, organisation of adult healthcare services and specific transition topics such as driving, drugs, alcohol, relationships, finances and living away from home [5, 19]. Usual care included regular appointments with their paediatric diabetes care team (i.e. endocrinologist, diabetes nurse or dietitian) and post transfer with their adult diabetes team (i.e. endocrinologist and as-needed visits with a diabetes educator and/or a dietitian). All usual care services (i.e. clinic visits) are publicly supported by the province's universal healthcare system. Collaboration between paediatric and adult diabetes clinics to help with transition of care has been in place for at least two decades. An electronic transition checklist was used by all the paediatric clinics to ensure transition topics were covered prior to transfer in both groups. For both usual care and intervention groups, the paediatric referral letter to adult care included a transition care topic checklist and a summary of previous care by the paediatric multi-disciplinary team. Adult clinics provided receipt of referral to paediatric clinics and adults clinics contacted participants directly with a posted letter and a reminder call 1 week prior to initial and all followup appointments. These processes have been in place since 2008 and did not change during the study period.

The intervention group was provided additional support by way of a non-medical transition coordinator during the transition and transfer from paediatric to adult diabetes care. Prior to transfer, the transition coordinator met each participant in person once during their routine paediatric diabetes appointment to explain their role. The transition coordinator's role included the following tasks: (1) use of text messaging, email or telephone communication (as per participant's preference) to maintain contact with the participant every 2 months for 12 months past the transfer date; (2) use of text messaging, e-mail or telephone as needed when participants reached out to them to answer any questions whereby the transition coordinator would provide direction; (3) notifying paediatric or adult diabetes teams of emergency visits or hospitalisations; (4) assisting participants with finding family physicians (if needed); (5) assisting with completion of financial assistance/disability/insurance forms; (6) addressing any stated psychosocial needs by relaying information on community support for participants and families; and (7) maintaining a private Facebook page and a transition website, which participants were encouraged to join and/or visit. Website contents included information on transition, adult diabetes care (i.e. location, contact numbers, what to expect in adult care) and diabetes resources as well as mental health and resources. The transition coordinator did not provide any medical advice, counselling or assessment of psychosocial needs. The nonmedical transition coordinator had a university degree (Bachelor of Arts) and shadowed in our clinics for 2 months prior to study start. The transfer date was defined as the date of the referral to adult diabetes care. Twelve months after this date, a letter was sent to participants' attending adult endocrinologist and family physician to inform them that the transition coordinator's support was ending. Blinding of our intervention was not possible due to the nature of our intervention. No incentives were provided to participants or to any care providers.

Data sources The following data sources were used: (1) Electronic Medical Record for age, sex, PHN and date of transfer; (2) National Ambulatory Care Reporting System (NACRS) for claims data on ambulatory care appointments and emergency department visits [20]; (3) Discharge Abstract 
Database (DAD) for hospitalisations (coded using the ICD-10 CA diagnostic codes [http://apps.who.int/classifications/ icd10/browse/2016/en]); and (4) Alberta Health Services' provincial laboratory electronic repository. The data sources were linked using a personal unique lifetime identifier, the PHN, or participant postal code and were used to abstract data on clinical characteristics, sociodemographic factors and outcomes. The Pampalon Material Deprivation Index was used as a proxy for socioeconomic status, based on postal codes of participants [21]. These data sources have established reliability, robustness and ongoing quality assurance [22-26].

Outcomes Primary and secondary outcomes were defined a priori and were judged to be not open to misclassification or misinterpretation. The primary outcome was the proportion of participants that did not attend at least one routine clinic visit in adult diabetes care with a healthcare professional (i.e. endocrinologist, diabetes nurse or dietitian) within 1 year after transfer, as ascertained from NACRS claims data. Loss to follow-up was the primary outcome that has been used by three recent transition trials (13-15). Secondary outcomes included the following: mean total number of clinic visits with any healthcare professional collected from NACRS claims data; diabetes-related emergency department visits and hospitalisations for diabetic ketoacidosis collected from NACRS claims data or DAD, respectively; and mean $\mathrm{HbA}_{1 \mathrm{c}}$ as well as mean albumin/creatinine ratio (ACR). For laboratory data, we used all available results 1 year prior to the transfer date or up to 18 months after transfer date, where appropriate, and data were directly abstracted from the provincial laboratory electronic repository. Finally, previously validated questionnaires, including the Diabetes Self-Efficacy questionnaire, Problem Areas in Diabetes questionnaire, Diabetes Quality of life for Youth questionnaire and a programme satisfaction questionnaire, were used as indicators of satisfaction with care and diabetes-related quality of life [27-31]. Secondary clinical outcomes were assessed at 12 and 18 months post transfer.

Sample size and statistical analyses Baseline characteristics were presented as means with SD for numerical variables and as proportions for categorical variables. We first compared the usual care group to the intervention group on baseline demographic and clinical characteristics; for any significant differences found, these were controlled for in subsequent analysis. Continuous data were evaluated with a $t$ test, and categorical data were evaluated with a $\chi^{2}$ test for comparison between the two groups. Mixed-effects regression models were used to look at group differences, controlling for baseline levels. A mixed model regression approach was also used to examine the pattern of follow-up visits for each cohort. The final analysis involved logistic regression modelling to determine the best predictor of having no visits in adult care in the 1 year post transfer, while controlling for sex, duration of diabetes, Pampalon Material Deprivation Index, days from transfer date to first appointment and care-related variables in the year prior to transfer. The analyses took all available data into account and no participant was excluded for having incomplete data. All analyses used SPSS software, version 25 (IBM Analytics, Armonk, NY, USA).

In 2012, prior to this study, of young adults transferred to adult care, $24.3 \%$ had no visits and only $9.6 \%$ had one visit in adult care in the year following transfer. Based on an average of 120 youth transferred from our paediatric diabetes clinic with 60-75\% referred within Calgary each year, sample size calculations specified that recruiting 71 participants per group would be sufficient for a two-sided test maintaining $80 \%$ power and assuming an $\alpha$ of 0.05 to show a difference of $20 \%$ between the two groups, a difference deemed clinically important by our team of clinicians, health services researchers and decision makers. Because loss to follow-up was our study's primary outcome, we did not increase the sample size for this.

Ethics This study was approved by our Ethics Board (REB141158). All participants provided written informed consent for questionnaires.

\section{Results}

Baseline characteristics At baseline, there were no differences in age, sex, duration of diabetes, mean $\mathrm{HbA}_{1 \mathrm{c}}$, mean ACR, number of ambulatory care visits, or Pampalon Material Deprivation Index in the 1 year prior to transfer between the usual care group $(n=102)$ and the intervention group $(n=$ 101) (Table 1).

Routine clinic visits In the year following transfer, $47.1 \%$ of young adults in the usual care group vs $11.9 \%$ of the young adults in the intervention group did not attend any outpatient diabetes appointment in adult care $(p<0.01$; Table 2$)$. The mean number of follow-up visits was higher in the intervention group vs usual care group at 12 and 18 months (Table 2). For individuals with one visit, $94.4 \%$ vs $100 \%$ saw a physician in the usual care group vs the intervention group, respectively $(p=0.73)$. For individuals with two or more visits, $49 \%$ of those visits were with a physician in the usual care group compared with $57 \%$ in the intervention group ( $p=0.33$ ).

There were no between-group differences in the mean $\mathrm{HbA}_{1 \mathrm{c}}$ prior to transfer nor any changes in mean $\mathrm{HbA}_{1 \mathrm{c}}$ at 12 and 18 months (Tables 1,3 ). The number of $\mathrm{HbA}_{1 \mathrm{c}}$ tests were higher in the intervention group at 18 months (Table 3 ). Overall emergency department visits and hospitalisations rates were low; we found no significant changes from baseline 
Table 1 Baseline characteristics

\begin{tabular}{|c|c|c|c|}
\hline Characteristic & $\begin{array}{l}\text { Usual care } \\
(n=102)\end{array}$ & $\begin{array}{l}\text { Intervention } \\
(n=101)\end{array}$ & $p$ value \\
\hline Female sex, $n(\%)$ & $53(52.0)$ & $48(47.5)$ & 0.6 \\
\hline Age, years & $18.6 \pm 0.4$ & $18 \pm 0.4$ & 0.2 \\
\hline Time since diabetes diagnosis, years & $8.9 \pm 4.4$ & $9.7 \pm 4.0$ & 0.2 \\
\hline Mean $\mathrm{HbA}_{1 \mathrm{c}}$ during the 1 year prior to transfer, $\mathrm{mmol} / \mathrm{mol}$ & 78 & 74 & \\
\hline $\mathrm{HbA}_{1 \mathrm{c}}$ during the 1 year prior to transfer, $\%$ & $9.3 \pm 1.8$ & $8.9 \pm 2.0$ & 0.2 \\
\hline $\begin{array}{l}\text { Proportion with mean } \mathrm{HbA}_{1 \mathrm{c}}>75 \mathrm{mmol} / \mathrm{mol} \text { during the } \\
1 \text { year prior to transfer, } \%\end{array}$ & 38.2 & 36.6 & 0.8 \\
\hline ACR during the 1 year prior to transfer, $\mathrm{mg} / \mathrm{mol}$ & $1.7 \pm 2.2$ & $3.1 \pm 9.9$ & 0.3 \\
\hline No. of ambulatory care visits 1 year prior to transfer ${ }^{\mathrm{a}}$ & $5.8 \pm 9.5$ & $7.1 \pm 7.0$ & 0.3 \\
\hline \multicolumn{4}{|l|}{ Pampalon Material Deprivation Index quintile, $n(\%)$} \\
\hline Quintile 1 (most affluent) & $32(31.4)$ & $30(29.7)$ & - \\
\hline Quintile 2 & $29(28.4)$ & $31(30.7)$ & - \\
\hline Quintile 3 & $15(14.7)$ & $18(17.8)$ & - \\
\hline Quintile 4 & $8(7.8)$ & $10(9.9)$ & - \\
\hline Quintile 5 (most deprived) & $18(17.6)$ & $12(12)$ & $0.4^{\mathrm{b}}$ \\
\hline
\end{tabular}

at 12 and 18 months and no differences between groups (Table 3).

There were no between-group differences in the proportion of participants with a mean $\mathrm{HbA}_{1 \mathrm{c}}>75 \mathrm{mmol} / \mathrm{mol}(9 \%)$ prior to transfer (36.6\% usual care group vs $38.2 \%$ intervention group, $p=0.8$; Table 1); however, there were significantly fewer participants with abnormally high mean $\mathrm{HbA}_{1 \mathrm{c}}$ values (i.e. $>75 \mathrm{mmol} /$ $\mathrm{mol})$ in the intervention group at 12 months after transfer, compared with the usual care group $(41.1 \%$ in the usual care group vs $24.8 \%$ in the intervention group, $p=0.01)$. This pattern remained at 18 months after transfer $(42.2 \%$ of the usual care group vs $25.7 \%$ of the intervention group, $p=0.01$ ).

The overall frequency of communication was $7.3 \pm 3.3$ (mean $\pm \mathrm{SD}$ ) per individual and differed by communication method $(p<0.001)$. Most individuals $(55 \%)$ preferred texting (mean \pm SD number of texts $8.0 \pm 3.2$ ) compared with e- mailing (43\%; mean $\pm \mathrm{SD}$ number of e-mails $6.5 \pm 3.5, p=$ $0.046)$ or telephoning (2\%; mean \pm SD number of calls $5.5 \pm$ 2.2, $p<0.001)$.

Results of mixed modelling showed differences in the pattern of follow-up visits for the two cohorts ( -2 log likelihood $=1392.86$ ). In particular, the prospective cohort had more visits during the first year as compared with the retrospective cohort $[F(1,1449)=93.46, p<0.001]$. The results of mixed modelling did not show clustering at a certain time $[F(1,692)=4.18, p=0.54]$.

Finally, a multivariate logistic regression predicting absence of follow-up within 12 months of transfer showed that participants in the usual care group were more likely to not attend follow-up in the year following transfer (Table 4).

There were low rates of questionnaire completion in both intervention (16\%) and control (12\%) groups. Results were
Table 2 Comparison of routine care visits

\begin{tabular}{|c|c|c|c|}
\hline Characteristic & $\begin{array}{l}\text { Usual care } \\
(n=102)\end{array}$ & $\begin{array}{l}\text { Intervention } \\
(n=101)\end{array}$ & $p$ value \\
\hline No follow-up visits in the 1 year after transfer, $\%(n)$ & $47.1(48)$ & $11.9(12)$ & $<0.01$ \\
\hline Only one visit in the 1 year after transfer, $\%(n)$ & $17.6(18)$ & $2.0(2)$ & $<0.01$ \\
\hline$\geq 2$ visits in the 1 year after transfer, $\%(n)$ & $35.3(36)$ & $86.1(87)$ & $<0.01$ \\
\hline No follow-up visits in the 18 months after transfer, $\%(n)$ & $47.1(48)$ & $9.9(10)$ & $<0.01$ \\
\hline $\begin{array}{l}\text { Total number of follow-up visits with any } \\
\text { healthcare professional }{ }^{\mathrm{a}} \text { in the } 1 \text { year after transfer, } \\
\text { mean } \pm \mathrm{SD}\end{array}$ & $3.0 \pm 5.9$ & $5.8 \pm 6.0$ & $<0.01$ \\
\hline $\begin{array}{l}\text { Total number of follow-up visits with any healthcare } \\
\text { professional }^{\mathrm{a}} \text { in the } 18 \text { months after transfer, mean } \pm \text { SD }\end{array}$ & $3.0 \pm 5.9$ & $6.0 \pm 6.0$ & $<0.01$ \\
\hline
\end{tabular}

${ }^{\mathrm{a}}$ Endocrinologist, diabetes educator or dietitian 
Table 3 Mixed-effects regression models for clinical variables, emergency department visits and hospitalisations for diabetic ketoacidosis

\begin{tabular}{|c|c|c|c|}
\hline Characteristic & $\begin{array}{l}\text { Usual care } \\
(n=102)\end{array}$ & $\begin{array}{l}\text { Intervention } \\
(n=101)\end{array}$ & $\begin{array}{l}p \text { value } \\
\text { for group }\end{array}$ \\
\hline Mean $\mathrm{HbA}_{1 \mathrm{c}}$ during the 1 year prior to transfer, $\mathrm{mmol} / \mathrm{mol}$ & 78 & 74 & - \\
\hline $\mathrm{HbA}_{1 \mathrm{c}}$ during the 1 year prior to transfer, $\%$ & $9.3 \pm 1.8$ & $8.9 \pm 2.0$ & - \\
\hline Mean $\mathrm{HbA}_{1 \mathrm{c}}$ during the 1 year after transfer, $\mathrm{mmol} / \mathrm{mol}$ & 77 & 73 & 0.2 \\
\hline $\mathrm{HbA}_{1 \mathrm{c}}$ during the 1 year after transfer, $\%$ & $9.2 \pm 1.8$ & $8.8 \pm 1.9$ & 0.2 \\
\hline Mean $\mathrm{HbA}_{1 \mathrm{c}}$ during the 18 months after transfer, $\mathrm{mmol} / \mathrm{mol}$ & 77 & 73 & 0.2 \\
\hline $\mathrm{HbA}_{1 \mathrm{c}}$ during the 18 months after transfer, $\%$ & $9.2 \pm 1.9$ & $8.8 \pm 1.9$ & 0.2 \\
\hline No. of $\mathrm{HbA}_{1 \mathrm{c}}$ measurements during the 1 year prior to transfer & $2.4 \pm 1.6$ & $2.8 \pm 1.2$ & - \\
\hline No. of $\mathrm{HbA}_{1 \mathrm{c}}$ measurements during the 1 year after transfer & $1.4 \pm 0.9$ & $1.5 \pm 1.2$ & 0.9 \\
\hline No. of $\mathrm{HbA}_{1 \mathrm{c}}$ measurements during the 18 months after transfer & $1.4 \pm 0.9$ & $2.2 \pm 1.5$ & $<0.01$ \\
\hline No. of diabetes ED visits during the 1 year prior to transfer & $0.2 \pm 0.5$ & $0.7 \pm 1.5$ & - \\
\hline No. of diabetes ED visits during the 1 year after transfer & $0.2 \pm 0.5$ & $0.4 \pm 1.4$ & 0.2 \\
\hline No. of diabetes ED visits during the 18 months after transfer & $0.2 \pm 0.5$ & $0.5 \pm 1.4$ & 0.2 \\
\hline No. of DKA episodes during the 1 year prior to transfer & $0.1 \pm 0.4$ & $0.1 \pm 0.2$ & - \\
\hline No. of DKA episodes during the 1 year after transfer & $0.1 \pm 0.3$ & $0.1 \pm 0.3$ & 0.1 \\
\hline No. of DKA episodes during the 18 months after transfer & $0.1 \pm 0.3$ & $0.1 \pm 0.3$ & 0.1 \\
\hline
\end{tabular}

Data are presented as mean $\pm \mathrm{SD}$, unless stated otherwise

DKA, diabetic ketoacidosis; ED, emergency department not analysed with this significant attrition in both groups despite telephone, e-mail and letter prompts.

\section{Discussion}

Our communication technology enhanced transition coordinator intervention was successful in improving clinic attendance in transitioning youth with type 1 diabetes. This is noteworthy as we demonstrated a fourfold improvement in loss to followup rates. The mean $\mathrm{HbA}_{1 \mathrm{c}}$ did not improve but the proportion of participants having their $\mathrm{HbA}_{1 \mathrm{c}}$ tested increased and the proportion with a mean $\mathrm{HbA}_{1 \mathrm{c}}>75 \mathrm{mmol} / \mathrm{mol}$ decreased in

Table 4 Multivariate logistic regression analysis for no visits in adult care 12 months post transfer

\begin{tabular}{ll}
\hline Characteristic & OR $(95 \% \mathrm{CI})^{\mathrm{a}}$ \\
\hline Usual care group & $4.9(1.8,13.8)$ \\
$\mathrm{Sex}^{\mathrm{b}}$ & $0.7(0.3,1.7)$ \\
Duration of diabetes & $1.0(0.9,1.1)$ \\
Pampalon Material Deprivation Index & $1.0(0.7,1.5)$ \\
$\begin{array}{l}\text { Number of diabetes-related ambulatory care } \\
\quad \text { visits during the 1 year prior to transfer }\end{array}$ & $0.8(0.7,0.9)$ \\
$\begin{array}{l}\text { Mean HbA } \\
\text { Number during the 1 year prior to transfer }\end{array}$ & $0.9(0.7,1.2)$ \\
$\quad$ department visits during the 1 year prior to transfer & $0.7(0.3,1.3)$ \\
Time from transfer date to first appointment, days & $1.0(1.0,1.0)$ \\
\hline${ }^{a}$ Model $\chi^{2}(8)=43.20, p<0.001$ & \\
${ }^{b}$ Male sex as reference group &
\end{tabular}

the intervention group. Diabetes-related emergency department visits and hospitalisations for diabetic ketoacidosis were low and occurred at the same rate in both groups during follow-up at 12 and 18 months.

There are several strengths to our study. First, we included the input of youth with diabetes and their families in the development of our intervention [8]. We felt that this was important in order to optimise our potential impact on outcomes, rather than relying on medical personnel feedback alone. Second, we demonstrated that a non-medical transition coordinator improved follow-up rates. Medical personnel such as diabetes educators and endocrinologists were available for diabetes care but participants were required to reach out to them personally for diabetes care. Conversely, the transition coordinator was easily accessible via text, e-mail or telephone and could assist them in navigating the adult world. Importantly, having non-medical personnel as our transition coordinator was safe as there was no increase in emergency department visits or hospitalisations. Finally, we used common communication technology (i.e. texting, e-mail, etc.) in our intervention and this was well accepted and embraced by participants. This technology is readily accessible, sustainable and universal to many other geographic locales making it attractive for future interventions. There are also limitations to our study. First, our intervention did not support those transferring to places out of our geographical locale. Second, although we demonstrated a fourfold decrease in loss to follow-up, we did not show a reduction in emergency department visits or hospitalisations. Notably, our study was not powered to do so; nevertheless, we did not appreciate any increases either. Third, although we saw an improvement in the proportion of 
individuals with at least one visit in the year after transfer, a single visit may not indicate successful transition. Fortunately, the intervention group was also more likely to attend two or more clinic visits. The difference, though, between number of follow-up visits at 12 vs 18 months is small and may reflect lack of a sustained effect of the intervention. Fourth, our nonblinded study design using a historical comparison group could be a possible source of bias, with care providers aware of this intervention potentially changing their clinical care practices. To address this, we did include a washout period between our two groups to avoid care providers 'holding on' to patients they felt may benefit from the intervention. However, the use of a randomised controlled trial design would have resulted in a selection bias by not including the most vulnerable youth, and it may have taken longer and needed a larger budget to complete. Fifth, there was an unexpected difference in the follow-up rates between the group used in our sample calculation and the usual care group of our current study. We explored possible demographic differences between these groups and there were none. Noteworthy though, understanding that the true rate may fall in between these two proportions, our sample size had $80 \%$ power to detect a difference of $15 \%$ with a confidence of $95 \%$. Therefore, the current results are still meaningful for clinical care. Finally, despite telephone, e-mail and letter prompts, the response rate to our questionnaires were very low in both groups and could not be analysed meaningfully.

Recent transition trials have had variable results in improving post-transfer follow-up rates [12-14]. Sequeira et al. assessed a transition intervention with diabetes-specific education, a transition website, case management and access to a newly developed young adult diabetes clinic [13]. Unfortunately direct comparisons were not possible as the control group who were enrolled from a different site and significant baseline differences [13]. Our intervention was similar to that of Sequeira et al's in that we also included a transition website and transition support but in contrast we used a non-clinical transition coordinator rather than a case manager with a medical background for transition support. An Australian group assessed the effect, compared with usual care, of an appointment manager (intervention) who was a medical specialist and was a point of contact between youth participants and adult clinics [14]. In the 12 months post transfer, the mean frequency of appointments attended in the intervention vs control groups did not differ. More recently, a Canadian study assessed the role of a medical transition coordinator compared with standard care in an intervention with 6 months in paediatric care and 12 months in adult care and a 12 month follow-up period [12]. During the intervention, the mean number of follow-up visits were higher in the intervention group and they experienced improved satisfaction with care, less diabetes-related distress, and less emotional burden compared with their baseline scores but these findings did not persist in the follow-up period [12]. In comparison, we found improvements in follow-up at 12 months and 18 months when comparing the intervention group with the usual care group but appreciably there was little difference in the number of visits at 12 and 18 months (i.e. post intervention) in our intervention group which may reflect a lack of a sustained effect. Like our study, the Canadian study transition coordinators used texting and e-mail but in contrast to our study, which used a non-clinical transition coordinator, it used Certified Diabetes Educators for transition coordinators. In doing so, there were significant differences in the roles of transition coordinators, with our transition coordinator not providing any medical support (i.e. insulin adjustments, sick day management, etc.) but providing information, possible supports and resources for participant's concerns, and encouragement for participants to use the information provided to them. The differing roles in our study may have had possible benefits for participants; perhaps because our transition coordinator was a non-medical person they were perceived as being more approachable, encouraging problem-solving and self-management skills, and being supportive in navigating the 'adult world'.

Although our study showed improvements in clinic attendance, overall glycaemic control and acute complications did not improve. There is conflicting literature on the effect of transition on glycaemic control, with some studies showing no change, some studies showing a deterioration and some showing improvement [32-34]. Three previous intervention studies in transition to date have not demonstrated improvements in glycaemic control [12-14]. In our study, the mean $\mathrm{HbA}_{1 \mathrm{c}}$ did not improve when compared with values prior to transition, nor when we extended follow-up to 18 months. Interestingly the proportion of those with $\mathrm{HbA}_{1 \mathrm{cs}}>75 \mathrm{mmol} /$ mol was lower in the intervention group at 12 months. This may be because of improved diabetes care (i.e. insulin adjustments, glucose monitoring) but also possibly because individuals lost to follow-up are likely to have higher $\mathrm{HbA}_{1 \mathrm{c}}$ values than those that remain in care [35]. We do not suspect this to be the reason in our study because there were no differences in testing rates 12 months post transfer. The numbers of diabetesrelated emergency department visits and hospitalisations for diabetic ketoacidosis were low prior to transfer and we were not expecting to find changes in our follow-up period as we were not powered to do so. We are interested to see if there is any impact on acute complications as we follow these groups longitudinally.

Our study has important findings and implications for clinicians and policymakers. We have successfully demonstrated a transition intervention in youth with type 1 diabetes that reduced loss to follow-up, a finding that is useful to healthcare organisations, healthcare providers, and patients and their families. Moreover, our intervention employed a nonmedical transition coordinator and the use of simple and 
readily accessible technologies may be easily transferable and sustainable in many environments. Use of a non-medical transition coordinator is beneficial as they can support youth with other chronic conditions (i.e. cystic fibrosis, congenital heart disease), not just one condition. From a broader healthcare perspective, employing a non-medical transition coordinator shared across multiple clinics could result in lower costs, which is attractive in the current environment of rising healthcare costs.

Future directions for research include following these groups longitudinally to better understand the impact of our intervention on outcomes long term. Other avenues for research would also be to explore strategies for those who leave home (i.e. for study, a gap year, etc.), potentially through technologies such as videoconferencing, apps or other internet-based approaches, as well as assessing our strategy in those transitioning with other chronic conditions. Furthermore, any future work exploring transition care should include patient experience as an important outcome to better understand factors at play.

In summary, our study demonstrated a successful intervention that bridges the gap for youth with type 1 diabetes transitioning between paediatric and adult care. We are encouraged by our findings but know that other strategies that minimise adverse health events and improve quality of care for youth with diabetes and their families through this phase of their lives are necessary. Our approach used a non-medical transition coordinator with simple, readily accessible communication technologies, which increases the transferability and sustainability of the intervention.

Acknowledgements We would like to thank our study participants and their families, our transition coordinator, the Alberta Children's Hospital and all diabetes care providers for their involvement in this study. We would like to thank R. Sigal (Departments of Medicine, Cardiac Sciences and Community Health Sciences, University of Calgary, Canada) for his review of the manuscript and thoughtful comments. Some of the data were presented as an abstract at the 55th EASD Annual Meeting in 2019.

Data availability The datasets generated during the current study are available from the corresponding author upon request.

Funding This work was funded by The Lawson Foundation (GRT 201440). The study sponsor/funder was not involved in the design of the study; the collection, analysis, and interpretation of data; writing the report; and did not impose any restrictions regarding the publication of the report.

Authors' relationships and activities The authors declare that there are no relationships or activities that might bias, or be perceived to bias, their work.

Contribution statement All authors conceived and designed the study. SC performed the data analysis. SB, SC and DP interpreted the data. SB, $\mathrm{SC}$ and DP wrote the manuscript. All authors edited the manuscript and approved the final version. SB and DP are the guarantors of this work and, as such, had full access to all the data in the study and takes responsibility for the integrity of the data and the accuracy of the data analysis.

\section{References}

1. Kipps S, Bahu T, Ong K et al (2002) Current methods of transfer of young people with type 1 diabetes to adult services. Diabet Med 19(8):649-654. https://doi.org/10.1046/j.1464-5491.2002.00757.x

2. Nakhla M, Daneman D, To T, Paradis G, Guttmann A (2009) Transition to adult Care for Youths with Diabetes Mellitus: Findings from a universal health care system. Pediatrics 124(6): e1134-e1141. https://doi.org/10.1542/peds.2009-0041

3. Gerstl E-M, Rabl W, Rosenbauer J et al (2008) Metabolic control as reflected by $\mathrm{HbA} 1 \mathrm{c}$ in children, adolescents and young adults with type-1 diabetes mellitus: Combined longitudinal analysis including 27,035 patients from 207 centers in Germany and Austria during the last decade. Eur J Pediatr 167(4):447-453. https://doi.org/10. 1007/s00431-007-0586-9

4. Hendricks M, Monaghan M, Soutor S, Chen R, Holmes CS (2013) A profile of self-care behaviors in emerging adults with type 1 diabetes. Diabetes Educ 39(2):195-203. https://doi.org/10.1177/ 0145721713475840

5. Peters A, Laffel L (2011) Diabetes care for emerging adults: Recommendations for transition from pediatric to adult diabetes care systems. Diabetes Care 34:2477-2485. https://doi.org/10. 2337/dc11-1723

6. Garvey KC, Telo GH, Needleman JS, Forbes P, Finkelstein JA, Laffel LM (2016) Health care transition in young adults with type 1 diabetes: Perspectives of adult endocrinologists in the U.S. Diabetes Care 39(2):190-197. https://doi.org/10.2337/dc15-1775

7. Garvey KC, Beste MG, Luff D, Atakov-Castillo A, Wolpert HA, Ritholz MD (2014) Experiences of health care transition voiced by young adults with type 1 diabetes: A qualitative study. Adolesc Health Med Ther 5:191-198. https://doi.org/10.2147/AHMT. S67943

8. Butalia S, McGuire KA, Dyjur D, Mercer J, Pacaud D (2020) Youth with diabetes and their parents' perspectives on transition care from pediatric to adult diabetes care services: A qualitative study. Health Sci Rep 3(3):e181. https://doi.org/10.1002/hsr2.181

9. Dabelea D, Mayer-Davis EJ, Saydah S et al (2014) Prevalence of type 1 and type 2 diabetes among children and adolescents from 2001 to 2009. JAMA 311(17):1778-1786. https://doi.org/10.1001/ jama.2014.3201

10. The Diamond Project Group (2006) Incidence and trends of childhood type 1 diabetes worldwide 1990-1999. Diabet Med 23(8): 857-866. https://doi.org/10.1111/j.1464-5491.2006.01925.x

11. Van Walleghem N, MacDonald CA, Dean HJ (2008) Evaluation of a systems navigator model for transition from pediatric to adult Care for Young Adults with Type 1 diabetes. Diabetes Care 31(8):1529-1530. https://doi.org/10.2337/dc07-2247

12. Spaic T, Robinson T, Goldbloom E et al (2019) Closing the gap: Results of the multicenter Canadian randomized controlled trial of structured transition in young adults with type 1 diabetes. Diabetes Care 42(6):1018-1026. https://doi.org/10.2337/dc18-2187

13. Sequeira PA, Pyatak EA, Weigensberg MJ et al (2015) Let's empower and prepare (LEAP): Evaluation of a structured transition program for young adults with type 1 diabetes. Diabetes Care 38 : 1412-1418. https://doi.org/10.2337/dc14-2577

14. White M, O'Connell MA, Cameron FJ (2017) Clinic attendance and disengagement of young adults with type 1 diabetes after transition of care from paediatric to adult services (TrACeD): A randomised, open-label, controlled trial. Lancet Child Adolesc Health 1(4):274 283. https://doi.org/10.1016/S2352-4642(17)30089-5

15. Ford I, Norrie J (2016) Pragmatic trials. N Engl J Med 375(5):454 463. https://doi.org/10.1056/NEJMra1510059

16. Elm E, Altman DG, Egger M, Pocock SJ, Gøtzsche PC, Vandenbroucke JP (2007) Strengthening the reporting of observational studies in epidemiology (STROBE) statement: Guidelines 
for reporting observational studies. BMJ 335:806-808. https://doi. org/10.1136/bmj.39335.541782.AD

17. American Diabetes Association (2013) Standards of medical Care in Diabetes - 2013. Diabetes Care 36(Supplement 1):S11-S66. https://doi.org/10.2337/dc13-S011

18. Canadian Diabetes Association Clinical Practice Guidelines Expert Committee (2013) CDA 2013 clinical practice guidelines for the prevention and Management of Diabetes in Canada. Can J Diabetes 37(suppl 31):S1-S212

19. McManus MA, Pollack LR, Cooley WC et al (2013) Current status of transition preparation among youth with special needs in the United States. Pediatrics 131(6):1090-1097. https://doi.org/10. 1542/peds.2012-3050

20. Canadian Institute for Health Information (2019) National Ambulatory Care Reporting System Metadata (NACRS). Available from https://www.cihi.ca/en/national-ambulatory-carereporting-system-metadata. Accessed 10 June 2019

21. Pampalon R, Hamel D, Gamache P, Raymond G (2009) A deprivation index for health planning in Canada. Chronic Dis Can 29(4): 178-191. https://doi.org/10.24095/hpcdp.29.4.05

22. Canadian Institute for Health Information (2019) Data and Information Quality. Available from https://www.cihi.ca/en/ submit-data-and-view-standards/data-and-information-quality. Accessed 10 June 2019

23. Butalia S, Johnson JA, Ghali WA, Rabi DM (2013) Clinical and socio-demographic factors associated with diabetic ketoacidosis hospitalization in adults with type 1 diabetes. Diabet Med 30(5): 567-573. https://doi.org/10.1111/dme.12127

24. Butalia S, Patel AB, Johnson JA, Ghali WA, Rabi DM (2014) Association between diabetic ketoacidosis hospitalizations and driving distance to outpatient diabetes Centres in adults with type 1 diabetes mellitus. Can J Diabetes 38(6):451-455. https://doi.org/ 10.1016/j.jcjd.2013.12.009

25. Butalia S, Patel AB, Johnson JA, Ghali WA, Rabi DM (2017) Geographic clustering of acute complications and Sociodemographic factors in adults with type 1 diabetes. Can $\mathrm{J}$ Diabetes 41(2):132-137. https://doi.org/10.1016/j.jcjd.2016.08. 224

26. Canadian Institute for Health Information (2019) Discharge Abstract Database Metadata (DAD). Available from https://www. cihi.ca/en/discharge-abstract-database-metadata. Accessed 10 June 2019

27. Wallston KA, Rothman RL, Cherrington A (2007) Psychometric properties of the perceived diabetes self-management scale (PDSMS). J Behav Med 30(30):395-401

28. Ingersoll G, Marrero D (1991) A modified qualtiy -of-life measure for youths: Psychometric properties. Diabetes Educ 17:114-118

29. Welch GJA, Polonsky W (1997) The problem areas in diabetes scale: An evaluation of its clinical utility. Diabetes Care 20:760 766. https://doi.org/10.2337/diacare.20.5.760

30. Hermanns N, Kulzer B, Krichbaum M (2006) How to screen for depression and emotional problems in patients with diabetes: Comparison of screening characteristics of depression questionnaires, measurement of diabetes-specific emotional problems and standard clinical assessment. Diabetologia 49:469-477. https://doi. org/10.1007/s00125-005-0094-2

31. Pacaud D, Stephure D, Trussell R (2005) Problems in transition from pediatric care to adult care for individuals with diabetes. Can J Diabetes 19:13-18

32. Johnston P, Bell PM, Tennet H, Carson D (2006) Audit of young people with type 1 diabetes transferring from paediatric to adult diabetic services. Pract Diab Int 23(3):106-108. https://doi.org/10. 1002/pdi.914

33. Helgeson VS, Reynolds KA, Snyder PR et al (2013) Characterizing the transition from paediatric to adult care among emerging adults with type 1 diabetes. Diabet Med 30(5):610-615. https://doi.org/10. 1111/dme.12067

34. Rollo A, Salardi S, Ciavarella A et al (2014) Transition from pediatric to adult care. Eight years after the transition from pediatric to adult diabetes care: Metabolic control, complications and associated diseases. J Endocrinol Investig 37(7):653-659. https://doi.org/10. 1007/s40618-014-0090-9

35. Lyons SK, Becker DJ, Helgeson VS (2014) Transfer from pediatric to adult health care: Effects on diabetes outcomes. Pediatr Diabetes 15(1):10-17. https://doi.org/10.1111/pedi.12106

Publisher's note Springer Nature remains neutral with regard to jurisdictional claims in published maps and institutional affiliations. 